\title{
O desafio da qualidade e integridade das publicações científicas
}

\author{
The challenge of the quality and integrity of scientific publications \\ El reto de la calidad y la integridad de las publicaciones científicas
}

Thiago Dias Sartia

\section{Resumo}

A crescente importância das ciências levanta questões relacionadas à validade, veracidade, relevância, utilidade, accountability e sustentabilidade dos produtos da prática científica. Portanto, as práticas que atentem para a integridade da pesquisa científica são fundamentais, tanto por parte dos pesquisadores quanto por parte da revistas científicas. Em 2016, a Revista Brasileira de Medicina de Família e Comunidade (RBMFC) recusou 14 trabalhos que apresentavam problemas de plagiarismo, o que representa aproximadamente $10 \%$ das submissões do ano. Consideramos este um sério problema, que exige maior atenção e cuidado por parte dos autores que pretendem contribuir com a revista. A política editorial da RBMFC preza pela qualidade de suas publicações, respeitando princípios éticos e de integridade da pesquisa científica aceitos internacionalmente.

\section{Abstract}

The increasing importance of the sciences raises issues related to the validity, veracity, relevance, usefulness, accountability and sustainability of the products of scientific practice. Therefore, practices that compromise the integrity of scientific research are fundamental, both by researchers and by scientific journals. In 2016, the Brazilian Journal of Family and Community Medicine (RBMFC) refused 14 papers that had problems with plagiarism, which represents approximately $10 \%$ of the submissions of the year. We consider this a serious problem that requires greater attention and care from authors who intend to contribute to the journal. The editorial policy of RBMFC values the quality of its publications, respecting internationally accepted ethical principles and integrity of scientific research.

\section{Resumen}

La creciente importancia de la ciencia plantea preguntas acerca de la validez, veracidad, relevancia, utilidad, responsabilidad y sostenibilidad de los productos de la práctica científica. En 2016, la Revista Brasileña de Medicina Familiar y Comunitaria (RBMFC) negó 14 artículos con problemas de plagio, lo que representa aproximadamente el $10 \%$ de las sumisiones del año. Consideramos que esto sea un problema grave que requiere una mayor atención y cuidado por los autores que deseen contribuir a la revista. La política editorial de RBMFC valora la calidad de sus publicaciones, respetando los principios de la integridad científica internacionalmente aceptados.

\author{
Palavras-chave: \\ Ética na Publicação Científica \\ Má Conduta Científica \\ Revisão por pares \\ Plágio
}

Keywords:

Scientific Publication Ethics

Scientific Misconduct

Peer Review

Plagiarism

Palabras clave:

Ética en la Publicación Científica Mala Conducta Científica Revisión por Expertos Plagio

Fonte de financiamento: declaram não haver. Parecer CEP: não se aplica. Conflito de interesses: declaram não haver. Procedência e revisão por pares: revisado por pares. Recebido em: 15/12/2016. Aprovado em: 15/12/2016. 
Vivemos em uma sociedade complexa, globalizada, desigual, profundamente reflexiva e de incertezas fabricadas, na qual se questiona a todo instante a capacidade do aparato técnico e científico de dar respostas concretas e efetivas aos inúmeros riscos produzidos pelo problemático processo de modernização e desenvolvimento econômico. ${ }^{1}$

A crescente importância, magnitude e densidade das ciências - em contraposição a um único campo científico homogêneo - coloca na pauta política, econômica, filosófica, cultural e científica questões relacionadas à validade, veracidade, relevância, utilidade, accountability e sustentabilidade dos saberes e produtos da prática científica. $^{2}$

Contudo, partindo do pressuposto de que a produção científica é fundamental para a abordagem dos problemas enfrentados pela sociedade - incluindo a saúde, o problema não reside apenas na validade e utilidade da produção científica. Em uma frequência alarmante, políticas de saúde são definidas sem a devida análise e incorporação do conhecimento científico disponível, ${ }^{3}$ o mesmo ocorrendo no cotidiano da atenção à saúde com a replicação de condutas e práticas sem respaldo em evidências científicas válidas e contextualizadas. ${ }^{4}$ Não basta produzir boa ciência. Ela precisa ser bem utilizada e a consolidação de sistemas e serviços de saúde depende disso. ${ }^{2}$

É neste sentido que devemos debater e instituir práticas que atentem para a qualidade, rigor e integridade na condução da pesquisa, bem como na divulgação da produção científica. Uma série de mecanismos sociais, econômicos e políticos fomentam a competição e o produtivismo acadêmico, induzindo pesquisadores a um intenso ritmo de publicação que pode comprometer a integridade da pesquisa. Isto pressiona as equipes de editores e revisores das revistas científicas a utilizarem ferramentas e fluxos que minimizem a possibilidade de publicação de artigos com graves erros, sem que, infelizmente, consigam alcançar pleno êxito. ${ }^{5}$

Dados mundiais referentes a artigos publicados são escassos e de difícil obtenção, mas estima-se uma taxa anual de 2,5 milhões de artigos publicados, com um crescimento anual de aproximadamente $3 \%$, o que representa uma movimentação financeira que alcança a casa dos bilhões de dólares. ${ }^{6}$

Por outro lado, em uma metanálise, $2 \%$ dos pesquisadores admitem ter fabricado, falsificado ou manipulado dados de pesquisa ao menos uma vez na vida e 33\% afirmam já terem adotado alguma prática questionável na condução de uma pesquisa. Quando questionados sobre a visão do pesquisador a respeito do comportamento de seus pares, estes percentuais sobem para aproximadamente $14 \%$ e $72 \%$, respectivamente. ${ }^{7} \mathrm{E}$ esta realidade preocupa ainda mais quando nos deparamos com o trabalho de loannidis, que aponta para a possibilidade de que a maior parte das pesquisas publicadas resulte em achados falsos, contraditórios ou desnecessários. ${ }^{8,9}$

Tal grau de crescimento e ao mesmo tempo insegurança em relação aos produtos da prática científica exige da comunidade acadêmica cuidados significativos com a qualidade do que se publica, tendo em vista alguns princípios basilares da integridade da pesquisa científica e acadêmica, tais como objetividade, honestidade, imparcialidade, independência e responsabilidade. ${ }^{10}$

Alguns trabalhos sugerem que, nos últimos anos, a taxa de retratação de artigos aumente acima do crescimento anual de artigos publicados, embora permaneça em níveis relativamente baixos (inferior a $1 \%){ }^{11,12}$ De difícil conceituação, más práticas científicas (aqui considerando comprometimento e manipulação da revisão por pares, fabricação e falsificação de dados e plagiarismo) lideram as estatísticas de retratação de artigos publicados. ${ }^{11}$ Em análise das retratações feitas em jornais da BioMed Central 
entre 2000 e 2015, observou-se que $76 \%$ destas decorrem de más práticas científicas, sendo 33\% por manipulação do processo de revisão por pares e $16 \%$ por plagiarismo. ${ }^{11}$

A gravidade do plagiarismo é de tal ordem que alguns episódios relatados podem chegar ao nível do inacreditável. Recentemente, Dansinger ${ }^{13}$ publicou uma carta na Annals of Internal Medicine denunciando que um artigo de seu grupo de pesquisa, submetido à Annals, havia sido roubado e publicado, com dados manipulados, por outro grupo em uma revista diferente alguns meses depois.

O que ocorreu? Dansinger e colaboradores submeteram um artigo referente a uma coorte realizada em Boston (EUA), sendo este recusado no processo de revisão por pares. Um dos pareceristas modificou alguns dados do artigo, incluindo a cidade e continente sede do trabalho, e publicou no EXCLI Journal, mantendo inalterado boa parte do texto, tabelas e figuras.

Além disso, o artigo foi publicado com diversos coautores, que autorizaram a inclusão de seus nomes sem terem nenhuma participação efetiva na pesquisa, sendo corresponsáveis pela conduta. $\mathrm{O}$ artigo foi retratado ${ }^{14}$ e a situação culminou em um editorial da Annals of Internal Medicine,${ }^{15}$ que frisou o impacto negativo que situações semelhantes provocam na reputação dos pesquisadores, nas suas instituições e na pesquisa científica em geral.

Outros problemas éticos relacionados à publicação científica incluem, com ou sem dolo dos pesquisadores, erros graves nos dados apresentados, duplicações de dados e imagens, ausência de aprovação ética do trabalho, falta de consentimento dos autores do artigo, conflitos de interesse não declarados e problemas de coautoria dos trabalhos. ${ }^{10}$ Cabe também acrescentar, por se tratar de questões da maior relevância para a Medicina de Família e Comunidade, as problemáticas e indevidas influências externas à pesquisa, induzindo conclusões que atendem interesses distantes da qualidade da pesquisa.

De janeiro ao início de dezembro de 2016, a Revista Brasileira de Medicina de Família e Comunidade (RBMFC) recebeu 142 submissões, sendo que 30 (31\%) foram aceitas para publicação. Das 85 submissões recusadas em 2016, 14 apresentavam problemas de plagiarismo, identificados com o auxílio do iThenticate ${ }^{\circledR}$, o que representa aproximadamente $10 \%$ das submissões e $16 \%$ das recusas do ano.

Consideramos este um sério problema, que exige maior atenção e cuidado por parte dos autores que pretendem contribuir com a revista. Observamos com frequência artigos que utilizam fragmentos de textos de outros trabalhos sem a devida citação da fonte original ou com citações equivocadas (o autor faz uma citação e o iThenticate ${ }^{\circledR}$ identifica aquele trecho como pertencendo a outra fonte não citada), em particular nas introduções e discussões dos artigos.

Além disso, em alguns artigos de revisão, os autores estruturam o texto com excessiva quantidade de citações diretas, sem a devida formatação conforme as normas de Vancouver adotadas pela RBMFC. O cuidado deve ser ainda maior quando se trata de artigos oriundos de trabalhos de conclusão de cursos de graduação ou especializações.

É importante destacar que o uso de programas específicos que identificam plágio é fundamental para as revistas científicas. Contudo, estes programas não são capazes de identificar todas as variações de plagiarismo, na medida em que a simples reprodução de textos de outras fontes sem a devida referência não contempla todo o problema. O uso de fragmentos de textos de outras fontes apenas com mudanças cosméticas das frases também é uma má prática científica. E o plagiarismo inclui a reprodução indevida de ideias de outros pesquisadores. Ciência se faz com reprodução de pesquisas nos mais diversos cenários 
sociais. Contundo, é desejável e ético que tais replicações sejam corretamente indicadas aos leitores e consumidores dos artigos e achados. ${ }^{5}$

A política editorial da RBMFC preza pela qualidade de suas publicações, respeitando princípios éticos e de integridade da pesquisa científica aceitos internacionalmente. O processo de revisão por pares é feito com o máximo de cuidado possível visando à menor probabilidade possível de ocorrência dos problemas citados acima. Conclamamos autores e colaboradores a juntos empreendermos esforços pela integridade da pesquisa científica. Os maiores beneficiários serão as pessoas que recebem nossos cuidados.

\section{Referências}

1. Beck U. Sociedade de risco: rumo a uma outra modernidade. São Paulo: Editora 34; 2010.

2. Greenhalgh T, Howick J, Maskrey N; Evidence Based Medicine Renaissance Group. Evidence based medicine: a movement in crisis? BMJ. 2014;348:g3725. DOI: http://dx.doi.org/10.1136/bmj.g3725

3. Brownson RC, Chriqui JF, Stamatakis KA. Understanding evidence-based public health policy. Am J Public Health. 2009;99(9):1576-83. DOI: http://dx.doi.org/10.2105/AJPH.2008.156224

4. Pope C. Resisting evidence: the study of evidence-based medicine as a contemporary social movement. Health. 2003;7(3):267-82. DOI: http://dx.doi.org/10.1177/1363459303007003002

5. Hames IM. Research integrity in an increasingly competitive and complex world. Beilstein Magazine. 2016;2(7). [citado 17 Jan 2017]. Disponível em: http://www.beilstein-institut.de/en/publications/magazine/scientific-essays/hames. DOI: http:// dx.doi.org/10.3762/bmag.7

6. Ware M, Mabe M. The STM Report. An overview of scientific and scholarly journal publishing. Hague: International Association of Scientific, Technical and Medical Publishers; 2015. [citado 17 Jan 2017]. Disponível em: http://www.stmassoc.org/2015_02_20_STM_Report_2015.pdf.

7. Fanelli D. How many scientists fabricate and falsify research? A systematic review and meta-analysis of survey data. PLoS One. 2009;4(5):e5738. DOI: http://dx.doi.org/10.1371/journal.pone.0005738

8. Ioannidis JP. Why most published research findings are false. PLoS Med. 2005;2(8):e124. DOI: http://dx.doi.org/10.1371/ journal.pmed.0020124

9. Ioannidis JP. The Mass Production of Redundant, Misleading, and Conflicted Systematic Reviews and Meta-Analyses. Milbank Q. 2016;94(3):485-514. DOI: http://dx.doi.org/10.1111/1468-0009.12210

10. European Science Foundation (ESF), All European Academies (ALLEA). The European Code of Conduct for Research Integrity. Strasbourg: ESF/ALLEA; 2011. P. 1-20. ISBN: 978-2-918428-37-4. [citado 17 Jan 2017]. Disponível em: http:// ec.europa.eu/research/participants/data/ref/h2020/other/hi/h2020-ethics_code-of-conduct_en.pdf

11. Moylan EC, Kowalczuk MK. Why articles are retracted: a retrospective cross-sectional study of retraction notices at BioMed Central. BMJ Open. 2016;6(11):e012047. DOI: http://dx.doi.org/10.1136/bmjopen-2016-012047

12. van Noorden R. Science publishing: The trouble with retractions. Nature. 2011;478(7367):26-8. DOI: http://dx.doi. org/10.1038/478026a

13. Dansinger M. Dear Plagiarist: A Letter to a Peer Reviewer Who Stole and Published Our Manuscript as His Own. Ann Intern Med. 2017;166(2):143. DOI: http://dx.doi.org/10.7326/M16-2551

14. Finelli C, Crispino P, Gioia S, La Sala N, D'amico L, La Grotta M, et al. Retraction: The improvement of large High-Density Lipoprotein (HDL) particle levels, and presumably HDL metabolism, depend on effects of low-carbohydrate diet and weight loss. EXCLI J. 2016;15:570.

15. Laine C. Scientific Misconduct Hurts. Ann Intern Med. 2017;166(2):148-9. DOI: http://dx.doi.org/10.7326/M16-2550

a Departamento de Medicina Social da Universidade Federal do Espírito Santo (UFES); Revista Brasileira de Medicina de Família e Comunidade (RBMFC). Vitória, ES, Brasil. tdsarti@gmail.com (Autor correspondente) 